\title{
Recent star formation in the Lupus clouds as seen by Herschel $\star, \star \star$
}

\author{
K. L. J. Rygl ${ }^{1}$, M. Benedettini ${ }^{1}$, E. Schisano ${ }^{1}$, D. Elia ${ }^{1}$, S. Molinari ${ }^{1}$, S. Pezzuto ${ }^{1}$, Ph. André ${ }^{2}$, J. P. Bernard ${ }^{3}$,
} G. J. White ${ }^{4,5}$, D. Polychroni ${ }^{6,1}$, S. Bontemps ${ }^{7,2}$, N. L. J. Cox ${ }^{8}$, J. Di Francesco ${ }^{9,10}$, A. Facchini ${ }^{1}$, C. Fallscheer ${ }^{9,10}$, A. M. di Giorgio ${ }^{1}$, M. Hennemann ${ }^{2}$, T. Hill $^{2}$, V. Könyves ${ }^{2}$, V. Minier ${ }^{2}$, F. Motte ${ }^{2}$, Q. Nguyen-Luong ${ }^{11}$, N. Peretto ${ }^{2}$, M. Pestalozzi ${ }^{1}$, S. Sadavoy ${ }^{9,10}$, N. Schneider ${ }^{7,2}$, L. Spinoglio ${ }^{1}$, L. Testi ${ }^{12,13}$, and D. Ward-Thompson ${ }^{14}$

1 Istituto di Astrofisica e Planetologia Spaziali (INAF-IAPS), via del Fosso del Cavaliere 100, 00133 Roma, Italy e-mail: kazi.rygl@inaf.it

2 Laboratoire AIM Paris-Saclay, CEA/IRFU CNRS/INSU Université Paris Diderot, 91191 Gif-sur-Yvette, France

3 CESR, Observatoire Midi-Pyrénées (CNRS-UPS), Université de Toulouse, BP 44346, 31028 Toulouse, France

${ }^{4}$ Rutherford Appleton LIbrary, Chilton, Didcot, OX11 0NL, UK

5 Department of Physics and Astronomy, Open University, Milton Keynes, UK

${ }^{6}$ University of Athens, Department of Astrophysics, Astronomy and Mechanics, Faculty of Physics, Panepistimiopolis, 15784 Zografos, Athens, Greece

7 CNRS/INSU, Laboratoire d'Astrophysique de Bordeaux UMR 5904, BP 89, 33271 Floirac, France

8 Instituut voor Sterrenkunde, KU Leuven, Celestijnenlaan 200D, 3001 Leuven, Belgium

9 Department of Physics and Astronomy, University of Victoria, PO Box 355, STN CSC, Victoria BC, V8W 3P6, Canada

10 National Research Council Canada, Herzberg Institute of Astrophysics, 5071 West Saanich Road, Victoria BC, V9E 2E7, Canada

11 Canadian Institute for Theoretical Astrophysics (CITA), University of Toronto, 60 St. George Street, Toronto, ON, M5S 3H8, Canada

12 European Southern Observatory, Karl-Schwarzschild-Strasse 2, 87548 Garching bei München, Germany

13 INAF-Osservatorio Astrofisico di Arcetri, Large E. Fermi 5, 50125 Firenze, Italy

14 Jeremiah Horrocks Institute, University of Central Lancashire, PR1 2HE, UK

Received 30 April 2012 / Accepted 16 November 2012

\section{ABSTRACT}

\begin{abstract}
We present a study of the star formation histories of the Lupus I, III, and IV clouds using the Herschel 70-500 $\mu$ m maps obtained by the Herschel Gould Belt Survey Key Project. By combining the new Herschel data with the existing Spitzer catalog we obtained an unprecedented census of prestellar sources and young stellar objects in the Lupus clouds, which allowed us to study the overall star formation rate (SFR) and efficiency (SFE). The high SFE of Lupus III, its decreasing SFR, and its large number of pre-main sequence stars with respect to proto- and prestellar sources, suggest that Lupus III is the most evolved cloud, and after having experienced a major star formation event in the past, is now approaching the end of its current star-forming cycle. Lupus I is currently undergoing a large star formation event, apparent by the increasing SFR, the large number of prestellar objects with respect to more evolved objects, and the high percentage of material at high extinction (e.g., above $A_{V} \approx 8 \mathrm{mag}$ ). Also Lupus IV has an increasing SFR; however, the relative number of prestellar sources is much lower, suggesting that its star formation has not yet reached its peak.
\end{abstract}

Key words. stars: formation - stars: protostars - ISM: individual objects: Lupus I - ISM: individual objects: Lupus III ISM: individual objects: Lupus IV - infrared: ISM

\section{Introduction}

In the current paradigm of low-mass star formation (SF), a gravitationally bound prestellar core will evolve into a young stellar object (YSO), passing through several phases, usually defined by Classes representing increasing stages of evolution: 0 , I, II, and III (see André et al. 2000; Lada \& Wilking 1984 for the definitions), before becoming a main-sequence star. While the later stages of low-mass SF are largely understood, less is known about the earlier stages (including the prestellar cores and the Class 0 objects) due to a lack of sensitivity and resolution at far-infrared to submm wavelengths. The Herschel Gould Belt

\footnotetext{
* Herschel is an ESA space observatory with science instruments provided by European-led Principal Investigator consortia and with important participation from NASA.

$\star \star$ Appendix A is available in electronic form at http://www . aanda.org
}

Survey (HGBS, André et al. 2010), carried out with the Herschel Space Observatory (Pilbratt et al. 2010), aims at studying these early stages of SF in nearby molecular clouds forming the socalled Gould Belt (Comerón et al. 1992).

Located at a distance between $150 \mathrm{pc}$ (Lup I and IV) and 200 pc (Lup III; Comerón 2008), the Lupus clouds (I, III, IV) are among the nearest star-forming regions in the Gould Belt. The large angular extent of the Lupus clouds across the sky $\left(334^{\circ}<l<352^{\circ}, 5^{\circ}<b<25^{\circ}\right)$ corresponds to a physical extent of $50 \mathrm{pc} \times 55 \mathrm{pc}$ at a distance of $150 \mathrm{pc}$, similar to the distance range among the Lupus I, III, and IV, clouds $(50 \mathrm{pc})$. Previous Spitzer (Merín et al. 2008, hereafter M08) and molecular-line (Benedettini et al. 2012) studies of Lupus I, III, and IV have found that the three clouds seem to be at different stages of evolution: Lupus I is thought to be the youngest cloud, Lupus IV is a little more evolved, and Lupus III is the most evolved cloud. 
The Lupus I, III, and IV clouds were mapped, as a part of the HGBS, at five wavelengths from $70 \mu \mathrm{m}$ to $500 \mu \mathrm{m}$, covering the range where the spectral energy distribution (SED) of cold dust emission from prestellar sources and envelopes of Class 0/I objects (protostars), is likely to peak. Therefore, the Herschel data are crucial for detecting these objects and determining their physical parameters. The combination of the M08 catalog, containing mostly Class II/III pre-main sequence (PMS) stars, with the prestellar cores and Class 0/I sources detected by Herschel allows for a much more complete view of the ongoing SF in the Lupus clouds than previously possible. Here, we present the first-look letter of the SF history of the Lupus clouds. A detailed analysis of the Herschel data on Lupus and the resulting cata$\log$ of identified objects will be presented in a forthcoming firstgeneration paper (Benedettini et al., in prep.) and will be publicly available at the HBGS website ${ }^{1}$.

\section{Herschel observations and data reduction}

The Lupus maps were obtained between January 2010 and January 2011 by photometric observations with the Photodetector Array Camera and Spectrometer (PACS; Poglitsch et al. 2010) and Spectral and Photometric Imaging Receiver (SPIRE; Griffin et al. 2010) in parallel mode using a scanning speed of $60^{\prime \prime} \mathrm{s}^{-1}$. Map sizes are $2^{\circ} \times 2.3$ for Lupus I and $1.5 \times 1.1$ for Lupus III, covering a similar region as the Spitzer observations for both clouds. Lupus IV was imaged in two maps, $2^{\circ} \times 1.3$ and $1.3 \times 1.3$, covering the Spitzer map, as well as a new region, never mapped before at wavelengths of 160-500 $\mu \mathrm{m}$. The data were exported from HIPE v 7.0 (Ott 2010) at level 0.5 , and processed with the ROMAGAL data reduction pipeline (Traficante et al. 2011; Piazzo et al. 2012). The maps were astrometrically aligned with the $70 \mu \mathrm{m}$ MIPS images from the Spitzer "cores2disks" (c2d) survey (Evans et al. 2003), which in turn have been aligned with 2MASS data, based on a number of point sources observed in both $70 \mu \mathrm{m}$ maps and yielding an astrometric precision of $\sim 2$ ". 5 . Absolute flux calibration (see Bernard et al. 2010) was found to be better than $20 \%$ by comparing the Herschel data with the Planck and IRAS data in the same area. The resulting maps have beam sizes of $9^{\prime \prime}, 12^{\prime \prime}, 18^{\prime \prime}, 25^{\prime \prime}, 36^{\prime \prime}$, for $70 \mu \mathrm{m}, 160 \mu \mathrm{m}, 250 \mu \mathrm{m}$, $350 \mu \mathrm{m}$, and $500 \mu \mathrm{m}$, respectively. The Lupus I observations were affected by stray Moonlight, visible as a bright band in the declination direction of the map. Fortunately, this did not affect our compact source fluxes, since the background is removed in the procedure.

For computing column density and temperature maps, the 70-350 $\mu \mathrm{m}$ maps were convolved to the $500 \mu \mathrm{m}$ resolution and

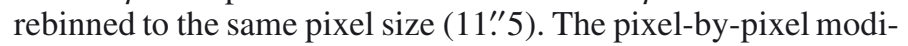
fied black-body fits were then performed on the regridded maps, excluding the $70 \mu \mathrm{m}$ map since this emission might not be tracing the cold dust exactly. For the modified black-body fitting we assumed a dust opacity of $\kappa_{1.3 \mathrm{~mm}}=0.004 \mathrm{~cm}^{2} \mathrm{~g}^{-1}$ (Hildebrand 1983), a grain emissivity parameter $\beta=2$, and the mean molecular weight $\mu=2.8$ (Kauffmann et al. 2008). The resulting column density map (Fig. A.1) was used to define the cloud area: the emission within the $A_{V}=2$ mag (assuming the column density to $A_{V}$ relation of $N_{\mathrm{H}_{2}}=9.4 \times 10^{20} A_{V} \mathrm{~cm}^{-2}$, Bohlin et al. 1978) contour was considered cloud emission and the integrated column densities in this contour (Table 1) agree within $20 \%$ with

\footnotetext{
1 http://gouldbelt-herschel. cea.fr/archives
}

Table 1. Properties of the Lupus clouds.

\begin{tabular}{|c|c|c|c|}
\hline Cloud & Lupus I & Lupus III & Lupus IV \\
\hline $\operatorname{distance}^{a}(\mathrm{pc})$ & 150 & 200 & 150 \\
\hline coverage $\left(\text { degree }^{2}\right)^{b}$ & 4.6 & 1.6 & 4.3 \\
\hline cloud area $>A_{V}=2 \mathrm{mag}\left(\mathrm{pc}^{2}\right)$ & 16.4 & 7.7 & 7.1 \\
\hline cloud mass $>A_{V}=2 \operatorname{mag}\left(M_{\odot}\right)$ & 830 & 570 & 500 \\
\hline cloud mass $>A_{V}=8 \mathrm{mag}\left(M_{\odot}\right)$ & 145 & 65 & 60 \\
\hline$N_{\text {tot }}=N_{\text {YSO }}+N_{\text {prestellar }}$ & 52 & 113 & 37 \\
\hline$N_{\text {YSO }} 0 / \mathrm{I} / \mathrm{II} / \mathrm{III}^{c}$ & $1 / 10 / 10 / 4$ & $1 / 10 / 50 / 42$ & $3 / 9 / 11 / 4$ \\
\hline$N_{\text {prestellar }}$ & 27 & 10 & 10 \\
\hline$N_{\text {unbound }}$ & 68 & 8 & 63 \\
\hline
\end{tabular}

Notes. ${ }^{(a)}$ See Comerón (2008) ${ }^{(b)}$ SPIRE and PACS overlapping area. (c) Number of objects per Class 0, I, II, and III are based on the Herschel data and on the M08 catalog.

the masses from the $\mathrm{c} 2 \mathrm{~d}$ extinction maps ${ }^{2}$ (Chapman et al. 2009) within the same area.

Compact source detection and extraction were performed with CuTEx (Molinari et al. 2011) in each of the five Herschel maps separately, using a $3 \sigma$ SNR detection limit. Following Elia et al. (2010), sources across the five bands were associated according to their positions. We adopted a conservative approach and removed sources that had a displaced counterpart in the $350 \mu \mathrm{m}$ or $500 \mu \mathrm{m}$ bands by more than half of the FWHP from the source center common to the other bands, which can introduce a large uncertainty in the measured fluxes at the longer wavelengths. In particular, for the prestellar cores this could result in finding false objects by an overestimation of the mass (see next section). Sources with detection in fewer than three bands longward of $\lambda=70 \mu \mathrm{m}$ were discarded. We then fit a single temperature modified black-body function to the SEDs of the extracted sources, again excluding the $70 \mu \mathrm{m}$ flux, to derive dust temperatures. Source masses were then derived from the optically thin part of the modified black-body spectrum, using the same dust properties as above. The reduced chisquared was used to remove sources with badly fitted SEDs, which would yield uncertain masses and temperatures otherwise, and hence could have a faulty prestellar core classification. Objects identified as non-Lupus members based on their proper motions (López Martí et al. 2011), in total 16 objects, were removed. We cross-checked our sample with the SIMBAD and the $\mathrm{c} 2 \mathrm{~d}$ database to remove known extragalactic sources (in total 13 galaxies were found). Seven off-cloud unresolved objects without a $70 \mu \mathrm{m}$ counterpart were classified as candidate galaxies and removed. Finally, to understand whether some of the off-cloud candidate protostars might have been misclassified (see Fig. A.1 and Sect. 4), we estimated the galaxy count completeness. Using the number counts from Clements et al. (2010), we find that for Lupus I and III we start lacking galaxies with flux levels of $\sim 350 \mathrm{mJy}$ at $250 \mu \mathrm{m}$, while for Lupus IV the limit is $700 \mathrm{mJy}$.

\section{Results}

Figure A.1 shows that the Lupus clouds are overall elongated in shape and fragmented into smaller clumps, in which most of the pre- and protostellar objects are located. A prestellar

\footnotetext{
2 http://data.spitzer.caltech.edu/popular/c2d/ 20071101_enhanced_v1/
} 
K. L. J. Rygl et al.: Recent star formation in the Lupus clouds as seen by Herschel

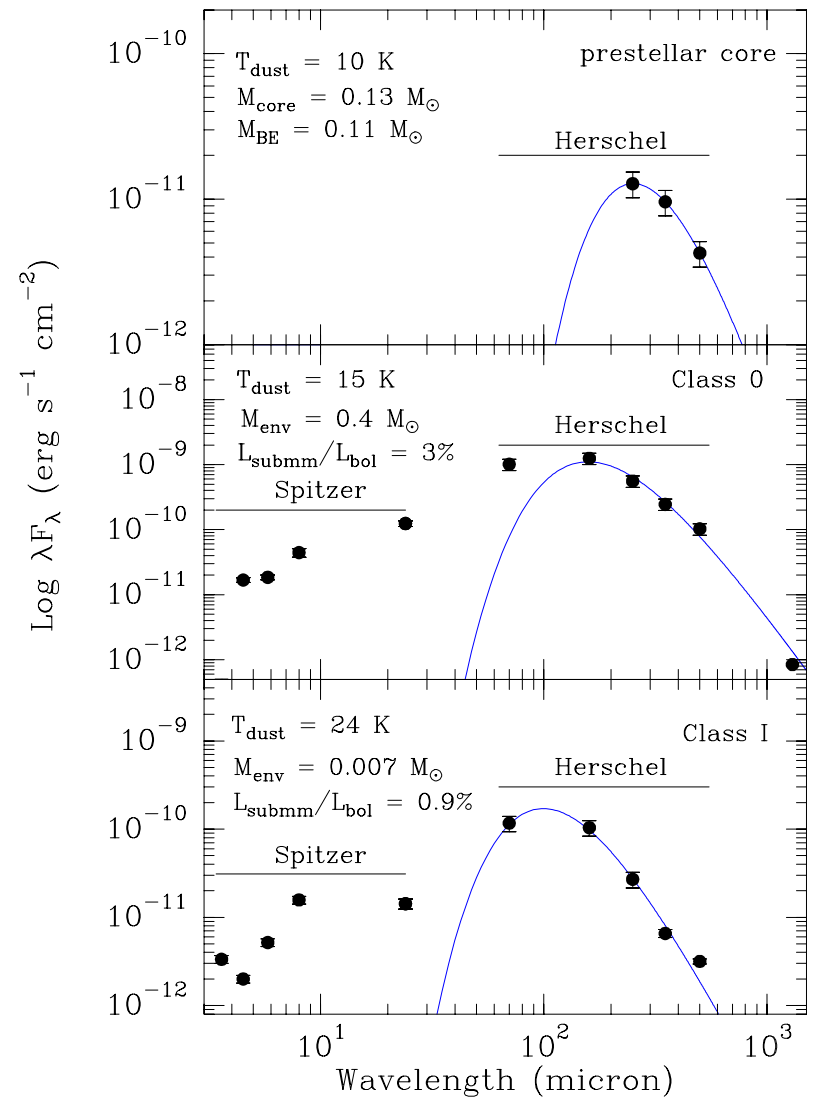

Fig. 1. Spectral energy distributions of a prestellar core, Class 0, and a Class I source in Lupus I and the modified black-body fit to the cold dust emission (blue line). The 3.4-24 $\mu \mathrm{m}$ and $1300 \mu \mathrm{m}$ data points were taken from M08.

core is defined as a gravitationally bound dense core (size $<0.05 \mathrm{pc}$ ) without an internal luminosity source that will supposedly form stars in the future (see e.g., Ward-Thompson et al. 1994; di Francesco et al. 2007). We used the ratio of the core mass to its critical Bonnor-Ebert mass $\frac{M}{M_{\mathrm{BE}}}$, to distinguish between prestellar cores and unbound objects that may or may not form stars (cf. Könyves et al. 2010), where $M$ is the core mass, $M_{\mathrm{BE}}=2.4 R c_{\mathrm{s}}^{2} / G$ the Bonnor-Ebert mass (Bonnor 1956), $c_{\mathrm{s}}$ the sound speed $\left(c_{\mathrm{s}}=\sqrt{k_{\mathrm{B}} T / \mu \mathrm{m}_{\mathrm{H}}}\right), R$ the radius, and $T$ the core temperature. For the core mass, we used the mass obtained from the modified black-body fitting and computed the $M_{\mathrm{BE}}$ using the fitted modified black-body temperature and the deconvolved radius at $250 \mu \mathrm{m}$. Cores with $\frac{M}{M_{\mathrm{BE}}}>1.0$ are gravitationally bound (Bonnor 1956) and were classified as prestellar, while cores with a lower ratio were considered unbound objects. An example of prestellar core SED is given in Fig. 1.

A protostar is defined as a core that is internally heated by an embedded YSO and is still accreting material from its envelope. The presence of a YSO is ascertained by a $70 \mu \mathrm{m}$ and/or $24 \mu \mathrm{m}$ source (Könyves et al. 2010). Among the protostellar objects, the submm to bolometric luminosity ratio (André et al. 2000), $L_{\mathrm{s} 2 \mathrm{~b}}=L_{\text {submm }}^{\lambda \geq 350 \mu \mathrm{m}} / L_{\mathrm{bol}}^{\lambda \geq 3.4 \mu \mathrm{m}}$, derived from the Herschel, M08, and WISE data (Wright et al. 2010), was used to identify the protostars as Class 0 or Class I objects. Protostars with $L_{\mathrm{s} 2 \mathrm{~b}} \geq 3 \%$ were classified as candidate Class 0 , while protostars with a lower ratio were identified as candidate Class I (a more conservative Class 0 definition than used by Bontemps et al. 2010). Examples of a Class 0 and a Class I SED are shown in Fig. 1. The classification of more evolved objects, i.e., the PMS stars (or
Class II/III objects), was taken from M08 and SIMBAD. M08 also identified flat SED objects that trace the transition between Class I and II (Greene et al. 1994). Since we cannot distinguish between Class I and F sources with the Herschel data, we considered them as Class I objects.

With the conservative source selection (Sect. 2) and the classification described above, we found 47 candidate prestellar cores (see Fig. 2, continued in Fig. A.2, for all the five-band images). For the entire Lupus complex, the prestellar cores have median masses of $0.25 M_{\odot}$ within a range of $0.1-3.0 M_{\odot}$, and median temperatures of $9 \mathrm{~K}$ within a range of $7-14 \mathrm{~K}$. The mean prestellar source size is $0.02 \pm 0.01 \mathrm{pc}$. Five Class 0 sources were found (Figs. 2 and A.2), of which most were previously classified as Class I objects (M08). The only previously known Class 0 is Lup MM3 (Tachihara et al. 2007). The Class 0 temperatures are between $9.5 \mathrm{~K}$ and $15 \mathrm{~K}$. Their envelope masses ranged from 0.08 to $0.6 M_{\odot}$. For two Class 0 sources, we compared the dust temperatures obtained from the Herschel data to the kinetic temperatures obtained from ammonia (Benedettini et al. 2012), and found that they agree within $20 \%$. We found 14 new Class I candidates in the Lupus clouds, 11 of which were outside the field covered by Spitzer c2d.

Table 1 summarizes the properties of the Lupus clouds and the objects found therein based on our results and the M08 catalog. The overlap between the Herschel data, including the YSOs that were only detected at $\lambda=70 \mu \mathrm{m}$ and $160 \mu \mathrm{m}$, with the M08 catalog was quite large, especially for the younger Class I objects. We detected $61 \%$ of their Class I objects, $46 \%$ of their Class II objects, and $10 \%$ of their Class III objects in the area covered by both surveys. For the few undetected Class I objects, we place an upper limit of $66 \mathrm{mJy}$ at $250 \mu \mathrm{m}$, and assuming a point source morphology, this implies a mass limit of $0.002 M_{\odot}$ at a dust temperature of $15 \mathrm{~K}$.

\section{Star formation history}

Palla \& Stahler (2000) posit that the star formation rate (SFR) has been increasing in the Lupus clouds over the past $4 \mathrm{Myr}$ using number counts of PMS stars, whose ages they estimated through comparison with theoretical evolutionary PMS tracks. Our sample contains both prestellar cores and Class 0/I objects, and by merging with the M08 catalog we can, therefore, estimate the recent SFR behavior better than in previous studies.

One can deduce the relative number of objects $N$ of a certain class with respect to a reference class expected for a constant SFR as $N=N_{\text {ref }} \times \tau / \tau_{\text {ref }}$ by assuming the classes' lifetimes $\tau$ and $\tau_{\text {ref }}$. Since the Class II objects are expected to be complete (M08 and SIMBAD), we chose this as the reference class with the lifetime estimate of $2 \pm 1 \mathrm{Myr}$ (see Evans et al. 2009). For the other classes, we assume $0.5 \mathrm{Myr}$ for the prestellar lifetime (Enoch et al. 2008), 0.05 Myr for the Class 0 lifetime (Froebrich et al. 2006), and 0.84 Myr for the Class I lifetime (Evans et al. 2009). Using these lifetimes, we derived the expected numbers of objects in each Class (relative to the number of Class II objects). Figure 3 shows the ratio $\eta$ of the observed-to-expected source numbers for the prestellar $\left(\eta_{\text {pres }}\right)$, Class $0\left(\eta_{\mathrm{Cl} 0}\right)$, and I $\left(\eta_{\mathrm{ClI}}\right)$ objects in each cloud. In this figure, we also plot the $\eta_{\mathrm{ClI}}$ without the possible galaxy contaminated sources (see Fig. A.1) based on the estimated completeness limit. Clearly, this possible contamination does not influence our SFR conclusions.

For both Lupus I and IV we find more prestellar, Class 0, and Class I objects than predicted for a constant SFR. We are therefore witnessing an increasing SFR over the past 0.5-1.5 Myr. The $\eta$ 's, particularly the $\eta_{\text {pres }}$, is much larger in Lupus I than in 

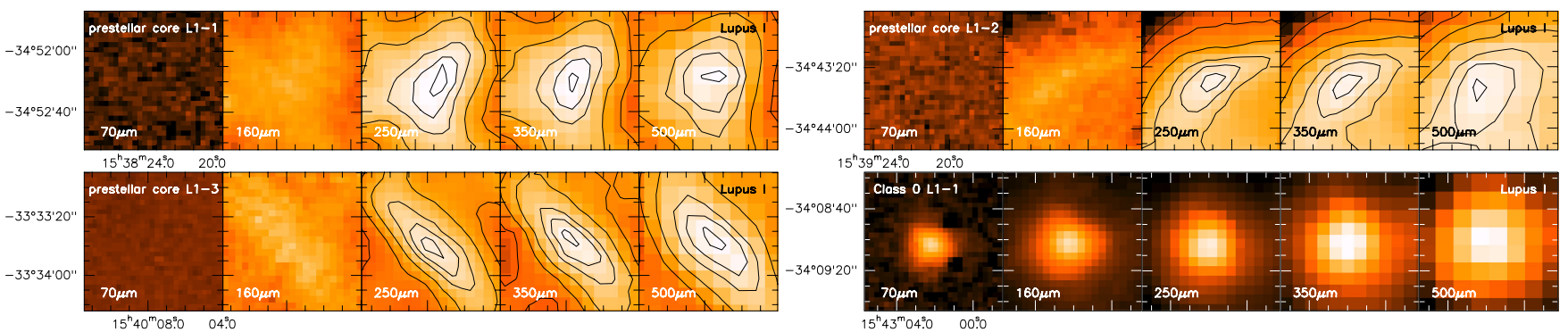

Fig. 2. Five-band images of three prestellar cores (contour levels are peakflux $* 0.99,0.95,0.90,0.80,0.70,0.60$, and 0.50 ) and a Class 0 object in Lupus I. The maps are centered on the prestellar core/Class 0 object and are ordered by right ascension. The remaining prestellar and Class 0 sources of all three Lupus clouds are given in Fig. A.2.

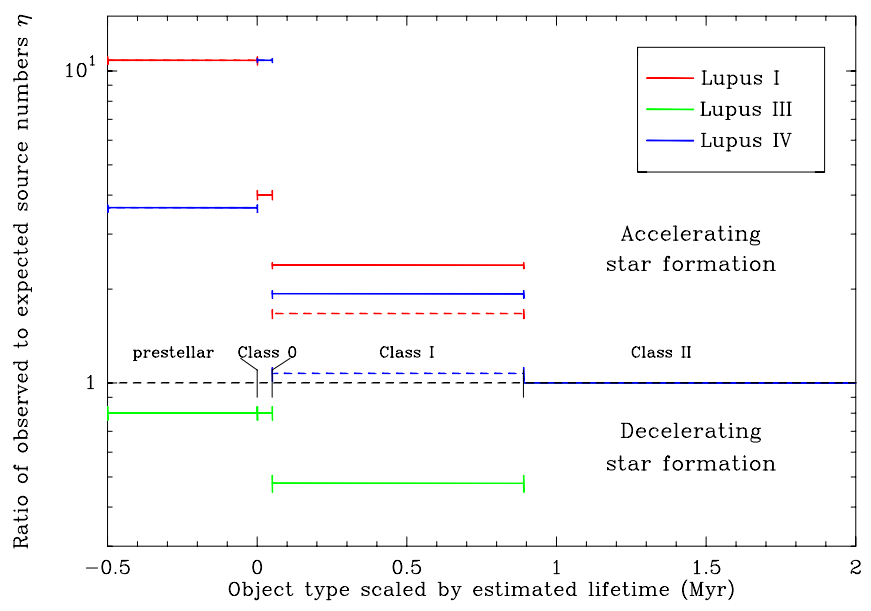

Fig. 3. Ratio of observed-to-expected (for a constant SFR) source numbers, $\eta$, per Class. Solid lines represent the numbers from Table 1, while dashed lines show Class I without the possible galaxy contamination.

Lupus IV, suggesting that Lupus I is undergoing a star formation event, while the SFR of Lupus IV might increase even more in the future. In Lupus III there are fewer prestellar, Class 0, and Class I objects than expected for constant SFR. The star formation in Lupus III has decelerated over the past 2 Myr.

The SFR analysis depends heavily on the lifetimes used in the estimation. Performing the inverse analysis, assuming a constant SFR, and deriving the lifetimes (with respect to the number of Class II objects) would lead to lifetimes longer by a factor $\sim 10$ for the prestellar sources, $\sim 4$ for the Class 0 objects, and $\sim 2$ for the Class I objects in Lupus I. Finally, the results of this analysis do not depend on the source-finding algorithm, CuTEx, since analysis of the sources found by getsources (Men'shchikov et al. 2012) yielded similar SFR trends.

We calculated the star formation efficiency (SFE), which is the ratio of the total mass in YSOs (Class 0-III) $M_{\mathrm{s}}$, assuming $0.2 M_{\odot}$ as the average Lupus PMS star mass (M08), to the total mass of the cloud plus YSOs: $\frac{M_{\mathrm{s}}}{M_{\text {cloud }}+M_{\mathrm{s}}}$. With this formulation, we find a similar SFE of $\sim 1 \%$ in Lupus I and IV, but a much higher SFE of 3.5\% in Lupus III. The latter may be expected from the high YSO-to-prestellar objects ratio (Table 1). The different distance of Lupus III, 200 pc, does not influence these results, because putting Lupus III at $150 \mathrm{pc}$ magnifies its SFE to $6.1 \%$.

One can study the star formation histories by interpreting the SFR behavior and SFE of the clouds as different evolutionary states within a star formation cycle. We propose that the high SFE of Lupus III, its decreasing SFR, and large number of PMS stars with respect to proto- and prestellar sources suggest that Lupus III is the most evolved cloud, which after having experienced a major star formation event, is now approaching the end of its current star-forming cycle. The properties of Lupus III seem to be similar to those of Chameleon I, where Belloche et al. (2011) claim to see the end of star formation based on prestellar cores found in $870 \mu \mathrm{m}$ LABOCA data. On the other hand, Lupus I is currently undergoing a large star formation event, apparent by the increasing SFR and the large number of prestellar objects with respect to more evolved sources. Also Lupus IV has an increasing SFR; however, the relative number of prestellar sources is much lower, suggesting that its star formation has not yet reached its peak and that Lupus IV is at an earlier stage of evolution than Lupus I. The contrast between the increasing SFR in Lupus I and the decreasing SFR in Lupus III is possibly reminiscent of the contrast between L1688 and L1689 in Ophiuchus. There, L 1688 is an active star-forming cloud with many prestellar cores, while L 1689 contains only a few prestellar cores, even though these clouds have similar CO properties. However, while Nutter et al. (2006) explain this difference by an external trigger from a nearby $\mathrm{OB}$ association, we claim that the diverse SFRs and SFEs in Lupus result from the clouds being in different states of their star formation cycle.

In Table 1 we also list the fraction of cloud mass above the SF threshold of $A_{V} \approx 8$ mag found in recent studies (e.g., Heiderman et al. 2010; André et al. 2010). This number, which is independent of source selection and identification, provides an estimate of the percentage of cloud mass directly available for SF, and it is expected to scale as the probability of finding prestellar cores. Spezzi et al. (2011) were the first to use this method on the extinction maps of the Lupus clouds. We applied this method to the Herschel data and found that Lupus I has a much higher percentage of mass above the $A_{V}$ threshold than the Lupus III and IV clouds, supporting the idea of a star formation event in this cloud. Lupus IV has a only slightly higher percentage of dense material than Lupus III, as was also noted by Spezzi et al. (2011), supporting the similar number of prestellar cores found in these two clouds. The fraction of cloud mass above this $A_{V}$ threshold correlates well with the number of prestellar cores found with Herschel, thereby strengthening our conclusions.

Acknowledgements. The authors thank the anonymous referee for her/his comments. SPIRE has been developed by a consortium of institutes led by Cardiff Univ. (UK) and including: Univ. Lethbridge (Canada); NAOC (China); CEA, LAM (France); IFSI, Univ. Padua (Italy); IAC (Spain); Stockholm Observatory (Sweden); Imperial College London, RAL, UCL-MSSL, UKATC, Univ. Sussex (UK); and Caltech, JPL, NHSC, Univ. Colorado (USA). This development has been supported by national funding agencies: CSA (Canada); NAOC (China); CEA, CNES, CNRS (France); ASI (Italy); MCINN (Spain); SNSB (Sweden); STFC, UKSA (UK); and NASA (USA). PACS has been developed by a consortium of institutes led by MPE (Germany) and including UVIE (Austria); KU Leuven, CSL, IMEC (Belgium); CEA, LAM (France); MPIA (Germany); 
K. L. J. Rygl et al.: Recent star formation in the Lupus clouds as seen by Herschel

INAF-IFSI/OAA/OAP/OAT, LENS, SISSA (Italy); IAC (Spain). This development has been supported by the funding agencies BMVIT (Austria), ESAPRODEX (Belgium), CEA/CNES (France), DLR (Germany), ASI/INAF (Italy), and CICYT/MCYT (Spain). KLJR, ES, DE, MP, and DP are funded by an ASI fellowship under contract numbers I/005/11/0 and I/038/08/0. NLJC is supported by the Belgian Federal Science Policy Office via ESA's PRODEX Program.

\section{References}

André, P., Ward-Thompson, D., \& Barsony, M. 2000, PPIV, 59

André, P., Men'shchikov, A., Bontemps, S., et al. 2010, A\&A, 518, L102

Belloche, A., Schuller, F., Parise, B., et al. 2011, A\&A, 527, A145

Benedettini, M., Pezzuto, S., Burton, M. G., et al. 2012, MNRAS, 419, 238

Bernard, J.-P., Paradis, D., Marshall, D. J., et al. 2010, A\&A, 518, L88

Bohlin, R. C., Savage, B. D., \& Drake, J. F. 1978, ApJ, 224, 132

Bonnor, W. B. 1956, MNRAS, 116, 351

Bontemps, S., André, P., Könyves, V., et al. 2010, A\&A, 518, L85

Chapman, N. L., Mundy, L. G., Lai, S.-P., \& Evans, N. J. 2009, ApJ, 690, 496

Clements, D. L., Rigby, E., Maddox, S., et al. 2010, A\&A, 518, L8

Comerón, F. 2008, Handbook of Star Forming Regions II, ed. B. Reipurth, 295

Comerón, F., Torra, J., \& Gomez, A. E. 1992, Ap\&SS, 187, 187

di Francesco, J., Evans, II, N. J., Caselli, P., et al. 2007, PPV, 17

Elia, D., Schisano, E., Molinari, S., et al. 2010, A\&A, 518, L97

Enoch, M. L., Evans, II, N. J., Sargent, A. I., et al. 2008, ApJ, 684, 1240
Evans, II, N. J., Allen, L. E., Blake, G. A., et al. 2003, PASP, 115, 965

Evans, II, N. J., Dunham, M. M., Jørgensen, J. K., et al. 2009, ApJS, 181, 321

Froebrich, D., Schmeja, S., Smith, M. D., et al. 2006, MNRAS, 368, 435

Greene, T. P., Wilking, B. A., Andre, P., et al. 1994, ApJ, 434, 614

Griffin, M. J., Abergel, A., Abreu, A., et al. 2010, A\&A, 518, L3

Heiderman, A., Evans, II, N. J., \& Allen, L. E., et al. 2010, ApJ, 723, 1019

Hildebrand, R. H. 1983, QJRAS, 24, 267

Kauffmann, J., Bertoldi, F., Bourke, T. L., et al. 2008, A\&A, 487, 993

Könyves, V., André, P., Men'shchikov, A., et al. 2010, A\&A, 518, L106

Lada, C. J., \& Wilking, B. A. 1984, ApJ, 287, 610

López Martí, B., Jiménez-Esteban, F., \& Solano, E. 2011, A\&A, 529, A108

Men'shchikov, A., André, P., Didelon, P., et al. 2012, A\&A, 542, A81

Merín, B., Jørgensen, J., Spezzi, L., et al. 2008, ApJS, 177, 551 (M08)

Molinari, S., Schisano, E., Faustini, F., et al. 2011, A\&A, 530, A133

Nutter, D., Ward-Thompson, D., \& André, P. 2006, MNRAS, 368, 1833

Ott, S. 2010, in ASP Conf. Ser., 434, 139

Palla, F., \& Stahler, S. W. 2000, ApJ, 540, 255

Piazzo, L., Ikhenaode, D., Natoli, P., et al. 2012, in IEEE, 21

Pilbratt, G. L., Riedinger, J. R., Passvogel, T., et al. 2010, A\&A, 518, L1

Poglitsch, A., Waelkens, C., Geis, N., et al. 2010, A\&A, 518, L2

Spezzi, L., Vernazza, P., Merín, B., et al. 2011, ApJ, 730, 65

Tachihara, K., Rengel, M., Nakajima, Y., et al. 2007, ApJ, 659, 1382

Traficante, A., Calzoletti, L., Veneziani, M., et al. 2011, MNRAS, 416, 2932

Ward-Thompson, D., Scott, P. F., Hills, R. E., et al. 1994, MNRAS, 268, 276

Wright, E. L., Eisenhardt, P. R. M., Mainzer, A. K., et al. 2010, AJ, 140, 1868

Pages 6 to 9 are available in the electronic edition of the journal at http: //www . aanda. org 


\section{Appendix A}
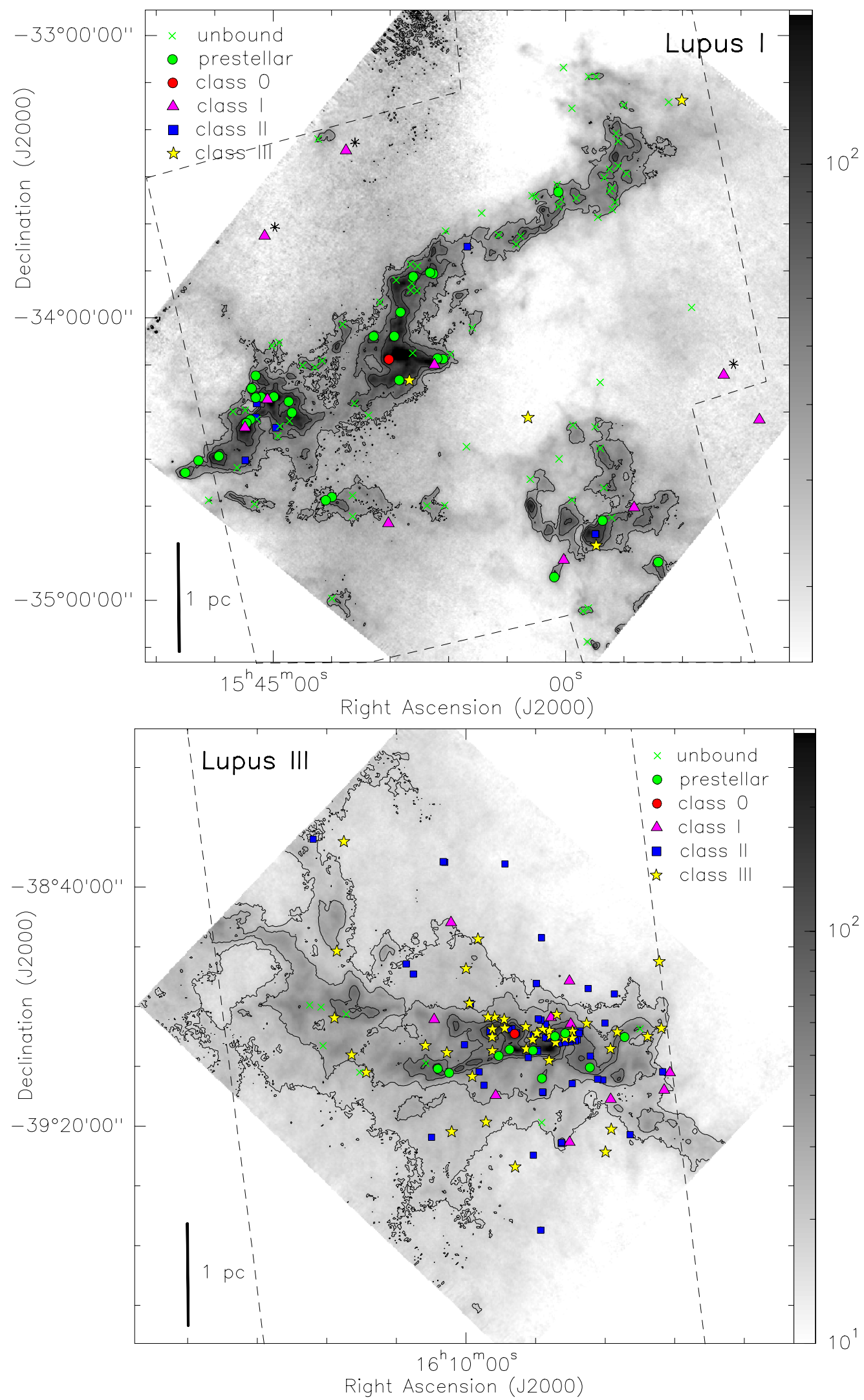

Fig. A.1. $\mathrm{H}_{2}$ column density maps of Lupus I, III, and IV in units of $10^{20} \mathrm{~cm}^{-2}$ with $A_{V}$ contours overplotted. For Lupus III and IV the contours are $A_{V}=2,3,6$, and $9 \mathrm{mag}$, while for Lupus I the contours are 4, 6, and 9 mag (to avoid the stray Moonlight). The different classes of objects, from the Herschel data and from the M08 catalog, are indicated. The off-cloud candidate Class I sources, marked by an asterisk, are those we considered as possible galaxy contaminations (shown by a dashed line in Fig. 3). The one off-cloud Class I in Lupus I without an asterisk has strong $24 \mu \mathrm{m}$ emission, so is less likely to be a galaxy. Dashed contours mark the Spitzer MIPS coverage. 
K. L. J. Rygl et al.: Recent star formation in the Lupus clouds as seen by Herschel

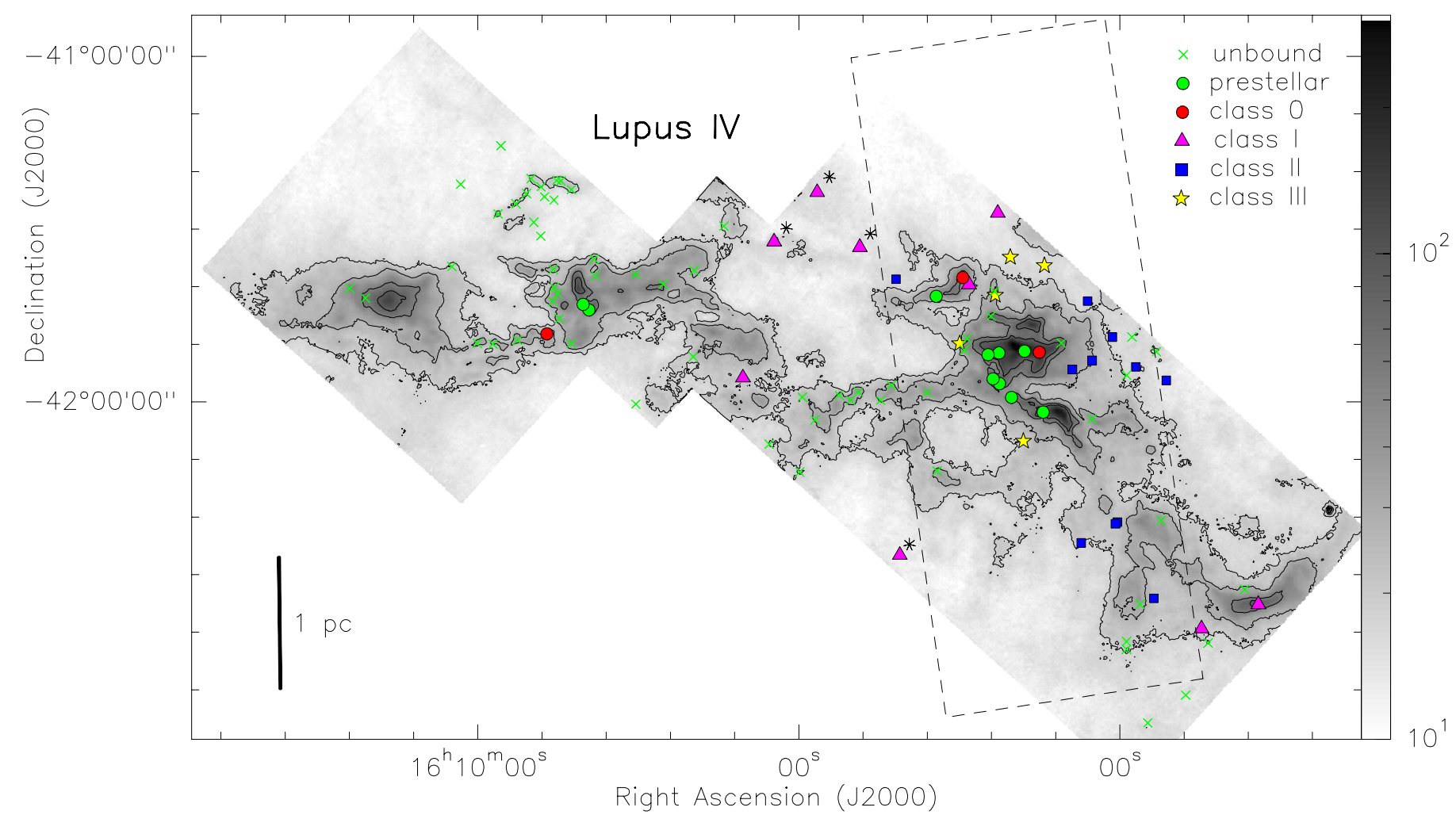

Fig. A.1. continued. 
A\&A 549, L1 (2013)
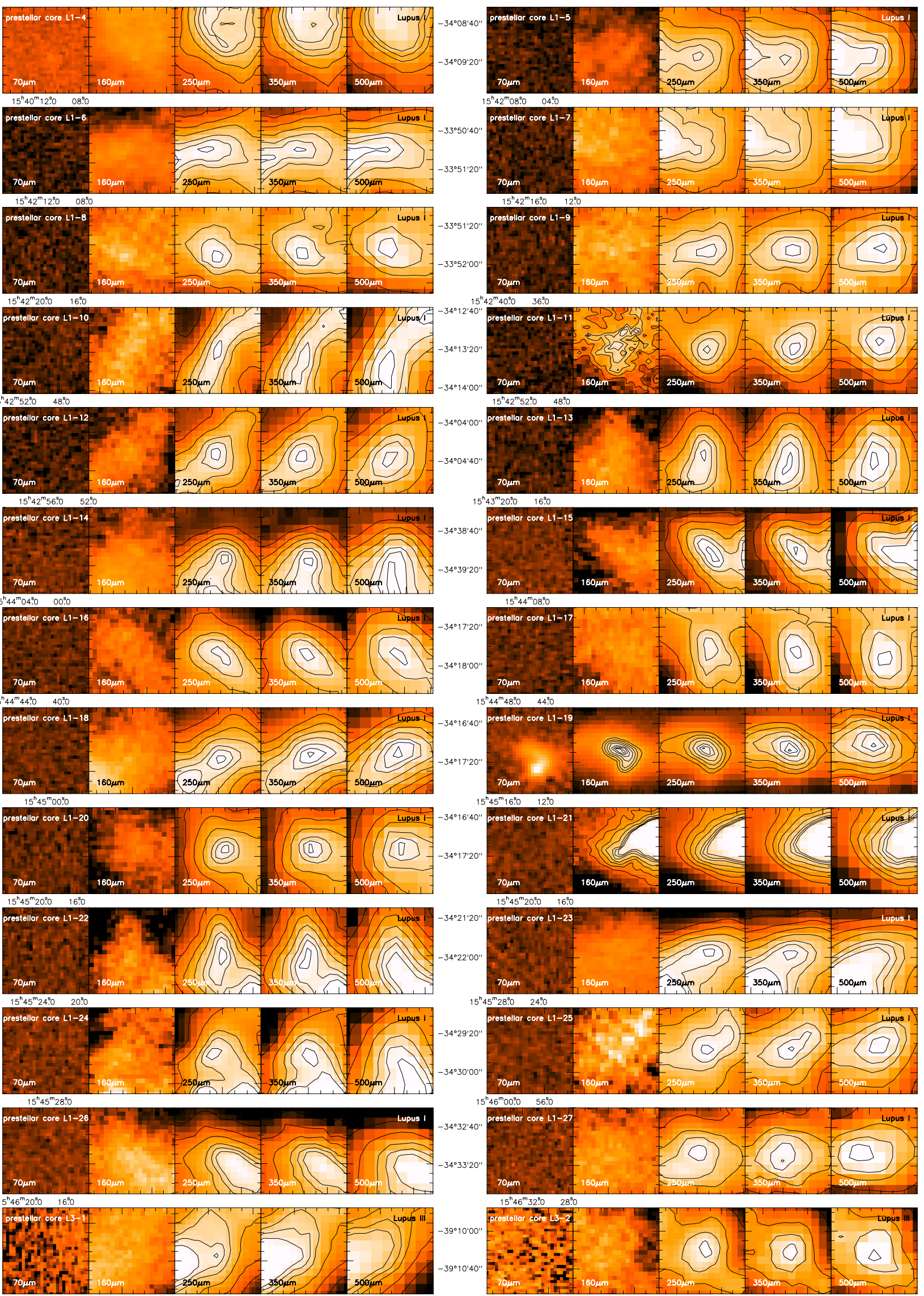

Fig. A.2. Visual catalog of the prestellar cores (contour levels are peakflux*0.99, 0.95, 0.90, 0.80, 0.70, 0.60, and 0.50 ) and Class 0 objects in the three Lupus clouds (I, III, IV), continued from Fig. 2. The maps are centered on the prestellar core/Class 0 object and are ordered per cloud by right ascension. 
K. L. J. Rygl et al.: Recent star formation in the Lupus clouds as seen by Herschel

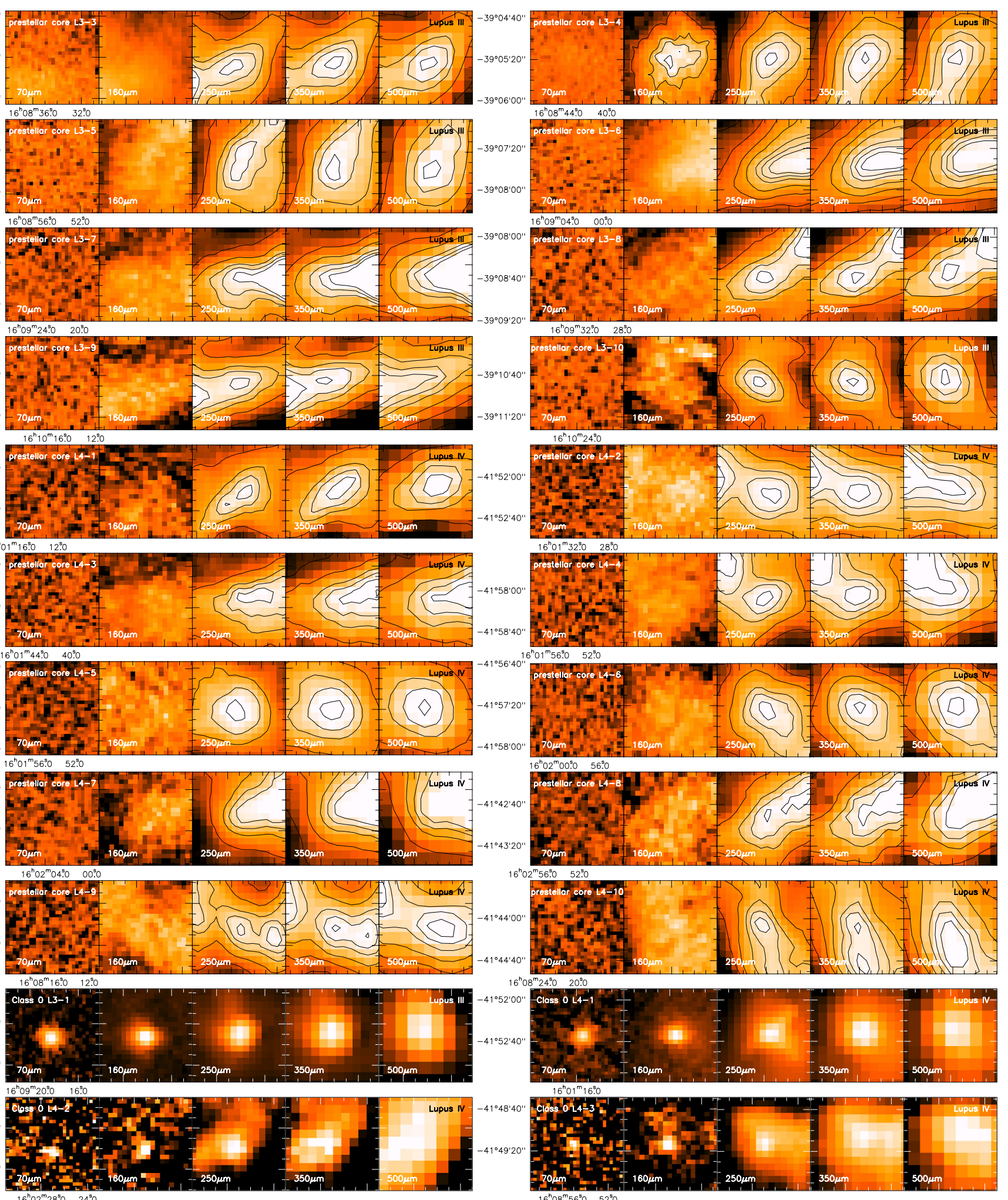

Fig. A.2. continued. 